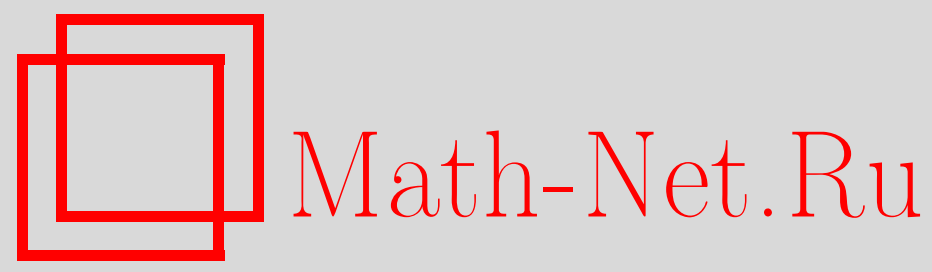

A. А. Грушо, Е. Е. Тимонина, Запреты в дискретных вероятностно-статистических задачах, Дискрет. матем., 2011, том 23, выпуск 2, 53-58

DOI: https://doi.org/10.4213/dm1140

Использование Общероссийского математического портала Math-Net.Ru подразумевает, что вы прочитали и согласны с пользовательским соглашением http://www . mathnet.ru/rus/agreement

Параметры загрузки:

IP : 54.224 .135 .184

26 апреля 2023 г., 08:34:55 


\title{
Запреты в дискретных вероятностно-статистических задачах
}

\author{
() 2011 г. А. А. Грушо, Е. Е. Тимонина
}

\begin{abstract}
Статья продолжает исследования, связанные с существованием состоятельных последовательностей критериев в дискретных вероятностно-статистических схемах.

В работе определено понятие «запрета» в дискретной вероятностной схеме и приведены примеры мер, в которых существуют «запреты».

В статистических задачах существуют критерии, критические множества которых полностью определяются через «запреты». Доказаны необходимые и достаточные условия существования состоятельных последовательностей критериев в дискретных схемах, в которых все критические множества определены через «запреты».

Работа выполнена при поддержке Российского фонда фундаментальных исследований, проект 10-01-00480.
\end{abstract}

\section{1. Введение}

Статья продолжает исследования авторов [1], связанные с существованием состоятельных последовательностей критериев в дискретных вероятностно-статистических схемах.

Вычисление статистических критериев в дискретных вероятностно-статистических схемах часто сводится к вычислению некоторых числовых характеристик этих последовательностей, например, рассматриваются частоты встречаемости отдельных знаков или комбинаций знаков. Вычислительная сложность таких критериев бывает довольно высокой. С целью упрощения вычислительной сложности таких критериев в этой работе мы рассматриваем «запреты» вероятностных мер, т.е. наличие комбинаций, обращающих в 0 все вероятности конечных поледовательностей, содержащих такие конфигурации.

В статье приведены примеры мер, в которых существуют «запреты».

В статистических задачах существуют критерии, критические множества которых полностью определяются через «запреты». Доказаны необходимые и достаточные условия существования состоятельных последовательностей критериев в дискретных схемах, в которых все критические множества определены через «запреты».

\section{2. Математическая модель}

Пусть $X=\left\{x_{1}, \ldots, x_{m}\right\}-$ конечное множество, $X^{n}-$ декартово произведение множества $X, X^{\infty}$ - множество бесконечных последовательностей с элементами из $X, \mathscr{A}-$ минимальная $\sigma$-алгебра, порожденная всеми цилиндрическими множествами (см. $[2,3,4])$. 
Если на $X$ рассматривается дискретная топология, то тихоновское произведение $X^{\infty}$ является компактным топологическим пространством со счетной базой $[3,4]$. В этом случае борелевская $\sigma$-алгебра $\mathscr{B}$ совпадает с $\sigma$-алгеброй $\mathscr{A}$.

Пусть $P_{0}-$ вероятностная мера на $\left(X^{\infty}, \mathscr{A}\right)$. Для каждого $n, P_{0, n}-$ проекция $P_{0}$ на первые $n$ координат последовательностей из $X^{\infty}$. Пусть $x-$ некоторый специальный символ, $\tilde{\omega}-$ последовательность элементов из $X$ конечной длины, $\omega-$ произвольная последовательность, $\omega \in X^{\infty}$.

Пусть последовательность $\widetilde{\omega}$ является подпоследовательностью $\omega$, начиная с заданного места. Назовем $\widetilde{\omega}$ вставкой в $\omega$ заданного вида, если $\widetilde{\omega}$ является подпоследовательностью $\omega$ с заданного места и структура подпоследовательности полностью определена. Если $\widetilde{\omega}=\left(\widetilde{\omega}_{1}, \ldots, \widetilde{\omega}_{k}\right)$, то структура вставки - это $\widetilde{\omega}$ и расстояния между элементами $\widetilde{\omega}$ в $\omega$, т.e.

$$
\omega=(\omega_{i_{1}}, \ldots, \omega_{i_{s}}, \widetilde{\omega}_{1}, \underbrace{}_{k_{1}}, \widetilde{\omega}_{2}, \underbrace{}_{k_{2}}, \ldots, \widetilde{\omega}_{k}) .
$$

Служебный символ $\varkappa$ помогает просто описать вставку вектора. Вставка $\tilde{y}-$ это вектор

$$
\tilde{y}=(\underbrace{\varkappa \ldots x}_{s}, \tilde{\omega}_{1}, \underbrace{\varkappa \ldots x}_{k_{1}}, \tilde{\omega}_{2}, \underbrace{\varkappa \ldots x}_{k_{2}}, \ldots, \tilde{\omega}_{k}, \ldots) .
$$

Определение 1. Вставка $\tilde{y}$ называется запретом в мере $P_{0, n}, n \geqslant s+\sum_{i=1}^{k} k_{i}$, если $P_{0, n}\left(\omega_{n}\right)=0$ для любого вектора $\omega_{n}$, содержащего вставку $\tilde{y}$.

Замечание 1. Пусть $A(\tilde{y}) \subseteq X^{n}-$ множество всех векторов с запретом $\tilde{y}$. Тогда для всех $n \geqslant n_{0}$ вероятность $P_{0, n}\left(A_{n}(\tilde{y})\right)=0$, начиная с некоторого $n_{0}$.

Для всякого множества $B_{n} \subseteq X^{n}$ очевидно равенство

$$
P_{0, n}\left(B_{n}\right)=P_{0}\left(B_{n} \times X^{\infty}\right) .
$$

Для любого $n$ существует носитель $D_{0, n}$ меры $P_{0, n}$ в $X^{n}$ или в $X^{\infty}$

$$
\Delta_{0, n}=D_{0, n} \times X^{\infty} .
$$

Легко видеть, что в $D_{0, n}$ входят все вектора $\omega_{n}: P_{0, n}\left(\omega_{n}\right)>0$.

Рассмотрим

$$
\bar{D}_{0, n}=X^{n} \backslash D_{0, n}
$$

Тогда для любого $\omega_{n} \in \bar{D}_{0, n}$ выполняется равенство

$$
P_{0, n}\left(\omega_{n}\right)=0
$$

Если $\omega_{n} \in X^{n}$ и $\omega_{k} \in X^{k}-$ два вектора, то $\omega_{n} \omega_{k}-$ конкатенация этих векторов, при этом $\omega_{n} \omega_{k} \in X^{n+k}$. Если для некоторого $n$ и $\omega_{n} \in X^{n}$ вероятность $P_{0, n}\left(\omega_{n}\right)=0$, то для каждого натурального $k$ и каждого $\omega_{k} \in X^{k}$ выполняется

$$
P_{0, n+k}\left(\omega_{n} \omega_{k}\right)=0 .
$$

В самом деле,

$$
P_{0, n+k}\left(\omega_{n} \omega_{k}\right)=P_{0}\left(\omega_{n} \omega_{k} \times X^{\infty}\right) \leqslant P_{0}\left(\omega_{n} \times X^{k} \times X^{\infty}\right)=P_{0}\left(\omega_{n} \times X^{\infty}\right)=0 .
$$

Эти рассуждения показывают, что при $\bar{D}_{0, n} \neq \varnothing$ для некоторого $n$ в последовательности $P_{0, n}$ существуют запреты. 


\section{3. Примеры запретов в вероятностных мерах на дискретных последовательностях}

Пример 1. Пусть $\omega_{2}, \ldots, \omega_{n}, \ldots$ - независимые одинаково распределенные случайные величины на $X=\{0,1\}$ с $p=1 / 2$ и $\omega_{1}=1$. Эта последовательность случайных величин порождает равномерную меру $P_{0}$ на $[1 / 2 ; 1]$, если каждую последовательность рассматривать как двоичную дробь. Тогда запретом является 0 на первом месте последовательности. Для любого $n$ вероятность $P_{0, n}\left(\omega_{n}\right)=0$ тогда и только тогда, когда $\omega_{n}$ начинается с нуля.

Рассмотрим две меры на $X^{\infty}$. Одна мера $P_{0}$ описана выше, вторая мера $P_{1}$ определяется так же, но $\omega_{1}=0$. Если мы проверяем гипотезу $H_{0}: P_{0}$ против альтернативы $H_{1}: P_{1}$, то наиболее мощный критерий строится на функции выявления запрета для $P_{0}$, т.е. лучший критерий определяется критическим множеством, зависящим только от запрета.

Пример 2. Пусть опять $X=\{0,1\}$. Рассмотрим простую однородную цепь Маркова с матрицей переходных вероятностей $\left(\begin{array}{ll}p & 1-p \\ q & 1-q\end{array}\right)$ и невырожденным начальным распределением. При $q=1$ запретом в этой цепи является вектор $(1,1)$, причем для любой последовательности $\omega_{n}$, в которой встречается этот вектор, $P_{0, n}\left(\omega_{n}\right)=0$. Из наличия этого вектора в любом фрагменте $\omega_{n}$ следует, что $P_{0, n}\left(\omega_{n}\right)=0$.

В этом случае также критерий проверки гипотезы $H_{0}$ : $0<p<1,0<q<1$, против альтернативы $H_{1}: 0<p<1, q=1$, может быть вычислен только на основании значения запрета. Алгоритм вычисления принадлежности к критическому множеству - это поиск запрета в наблюдаемой последовательности.

Запреты могут оказаться полезными в некоторых прикладных задачах, например, при выборе модели при анализе скрытых каналов [5].

Пример 3. Рассмотрим модель Симмонса [6] для скрытой передачи сигнала (сообщения) от $A$ к $B$, имеющими в распоряжении канал $T$ (сеть). Контролер $U$ перехватывает любые сообщения от $A$ к $B$ и решает, есть там скрытая передача или нет.

Пусть $X$ - множество возможных сообщений. Независимо от того, какими методами анализа обладает $U$, его решение строится на некотором множестве $S \subseteq X$ сообщений, которые $U$ принимает в качестве сообщений, содержащих скрываемую информацию. Если $x$ есть сообщение, наблюдаемое $U$, то $U$ принимает решение о существовании скрытой передачи в том случае, когда $x \in S$. Иными словами, у $U$ есть функция $\pi(x, S)$, вычислимая для него, где $\pi(x, S)$ - индикатор множества $S$. Если $x$ не принадлежит множеству $S$, то $U$ не считает $x$ нелегальной передачей, то есть не видит угрозы в $x$.

Рассмотрим вероятностную модель скрытого канала. Она предполагает существование вероятностной меры $P_{0}$ на $X$, соответствующей легальному выбору сообщения $x$. Иными словами, $U$ проверяет гипотезу $H_{0}: P_{0}$ по наблюдению $x$. Критерий задается критическим множеством $S$, отвергающим гипотезу $H_{0}$ с заданным уровнем вероятности ошибки $\varepsilon$ $\left(P_{0}(S) \leqslant \varepsilon\right)$.

Пусть участник $A$ предполагает, что модель легальной передачи в канале $T$ определяется распределением $P_{0}$. Передача в канале $T$ представляет собой бесконечную последовательность независимых одинаково распределенных случайных величин, принимающих значения в алфавите $X=\{0,1\}$ с вероятностями $p, q=1-p, p>0$. В рамках этой модели $A$ легко показывает, что следующая схема передачи абсолютно невидима для $U$.

Пусть множество сообщений участника $A$ есть $L_{1}, \ldots, L_{N}$. Он кодирует их следующим образом: строит $N$ случайных последовательностей $\Delta_{1}, \ldots, \Delta_{N}$ в соответствии с мерой $P_{0}$ и каждому сообщению $L_{i}, i=1, \ldots, N$, ставит в соответствие $\Delta_{i}, i=1, \ldots, N$. 
Кроме того, он строит случайную двоичную последовательность $G$. Для организации невидимого для $U$ канала эти данные скрытно для $U$ передаются участнику $B$. Пусть $A$ передал сообщение $L_{i}$. На местах, где в последовательности $G$ стоит $1, A$ дополнительно вставляет очередной знак последовательности $\Delta_{i}$. Таким образом, получена последовательность независимых одинаково распределенных случайных величин с $(p, q)$ распределением знаков на множестве $X$. В то же время, $U$ может видеть только последовательность независимых одинаково распределенных в соответствии с мерой $P_{0}$ случайных величин и не может видеть скрытой передачи.

Пусть, как и в предыдущем случае, $X=\{0,1\}$, и легальная передача порождается простой однородной цепью Маркова, в матрице которой вероятность перехода $P(1,1)=0$. Тогда скрытый канал предыдущего примера легко выявляется. В самом деле, в последовательности $\Delta_{i}, i=1, \ldots, N$, бесконечное число единиц, а вероятность того, что перед местами вписывания скрываемых знаков всегда будут нули, стремится к 0. Значит, с вероятностью, стремящейся к 1 , возникнет запрещенная в цепи Маркова комбинация $(1,1)$. При появлении такой комбинации $U$ однозначно выявляет скрытую передачу.

Пример 4. Продолжим рассмотрение примеров, связанных с запретами.

В традиции канала связи между двумя прикладными компьютерными системами, поток данных, как правило, структурирован или удовлетворяет правилам построения языковых форм. Случайность в таких последовательностях возникает при описании значений определенных параметров. Рассмотрим один из примеров такого структурирования.

Пусть сообщение содержит строку реляционного отношения, где формальная часть определена названиями атрибутов, за которыми следуют конкретные значения атрибутов. Вероятностная мера на передаваемой по каналу последовательности определяется случайной последовательностью значений атрибутов. Запреты определяются значениями атрибутов или наборов значений атрибутов, не входящие в домены значений этих атрибутов.

Пример 5. Запреты тесно связаны с методами имитозащиты и помехоустойчивыми кодами. В самом деле, при кодировании вводится избыточность, которая выражается в том, что определенная функция от участка рассматриваемой последовательности равна 0. В противном случае данная комбинация в мере исходного пространства сообщений (без учета шума) имеет вероятность 0.

\section{4. Использование запретов в статистике}

Рассмотрим опять измеримое пространство $\left(X^{\infty}, \mathcal{A}\right)$. Пусть на этом пространстве определены меры $P_{0}$ и $\left\{P_{\theta}, \theta \in \Theta\right\}$. Рассмотрим проекции этих вероятностных мер на первые $n$ координат, $n=1,2, \ldots$, которые будем обозначать $P_{0, n}$ и $\left\{P_{\theta, n}, \theta \in \Theta\right\}$. Носители этих мер обозначим через $D_{0, n}$ и $D_{\theta, n}$. Для любого $n$ и $B \subseteq X^{n}$ из согласованности проекций следует, что

$$
P_{0, n}(B)=P_{0}\left(B \times X^{\infty}\right), \quad P_{\theta, n}(B)=P_{\theta}\left(B \times X^{\infty}\right) .
$$

Для каждого $n, n=1,2, \ldots$, рассмотрим задачу проверки гипотезы $H_{0, n}: P_{0, n}$ против $H_{1, n}:\left\{P_{\theta, n}, \theta \in \Theta\right\}$. Для каждого $n$ критерий $T_{n}, n=1,2, \ldots$, определяется критическим множеством $S_{n}, n=1,2, \ldots$

Определение 2. Последовательность $T_{n}, n=1,2, \ldots$, критериев с критическими множествами $S_{n}$ называется состоятельной (СПК) [7] тогда и только тогда, когда

$$
P_{0, n}\left(S_{n}\right) \underset{n \rightarrow \infty}{\longrightarrow} 0,
$$


и для каждого $\theta \in \Theta$

$$
P_{\theta, n}\left(S_{n}\right) \underset{n \rightarrow \infty}{\longrightarrow} 1
$$

Определение 3. Критерий определяется запретами, если принадлежность статистических данных критическому множеству сводится к поиску запретов, известных для данного $n$.

Вообще говоря, о любом множестве можно также говорить, что оно определяется запретами, если в любой последовательности этого множества имеются запреты.

Очевидно, что для каждого $n$ множество $\bar{D}_{0, n}$ определяется запретами меры $P_{0, n}$. Нетрудно доказать, что носители меры $P_{0}$ и $P_{\theta}$ могут быть определены равенствами

$$
\begin{array}{ll}
\Delta_{0}=\lim _{n \rightarrow \infty} \Delta_{0, n}, & \Delta_{0, n}=D_{0, n} \times X^{\infty}, \\
\Delta_{\theta}=\lim _{n \rightarrow \infty} \Delta_{\theta, n}, & \Delta_{\theta, n}=D_{\theta, n} \times X^{\infty} .
\end{array}
$$

Теорема 1. Состоятельная последовательность критериев, все критические множества которой определяются запретами, существует тогда и только тогда, когда для каждого $\theta \in \Theta$ выполняется условие $P_{\theta}\left(\Delta_{0}\right)=0$.

Доказательство. Пусть в СПК для каждого $n$ критическое множество $S_{n}$ определяется запретами. Тогда $S_{n} \subseteq \bar{D}_{0, n}$. Обозначим $\Sigma_{n}=S_{n} \times X^{\infty}$. Тогда для каждого $n$

$$
\bar{D}_{0, n} \times X^{\infty} \cap \Delta_{0, n}=\varnothing .
$$

Последовательность множеств $\Delta_{0, n}$ не возрастает и, следовательно, для каждого $n$

$$
\bar{D}_{0, n} \times X^{\infty} \cap \Delta_{0}=\varnothing .
$$

Последовательность $\bar{\Delta}_{0, n}=\bar{D}_{0, n} \times X^{\infty}$, поэтому

$$
\Lambda=\lim _{n \rightarrow \infty} \bar{\Delta}_{0, n}=\bigcup_{n=1}^{\infty} \bar{\Delta}_{0, n} .
$$

Ясно, что $\Lambda \cap \Delta_{0}=\varnothing$. Из условия состоятельности следует, что для каждого $\theta \in \Theta$

$$
\lim _{n \rightarrow \infty} P_{\theta}\left(\Sigma_{n}\right)=1
$$

Тогда

$$
\lim _{n \rightarrow \infty} P_{\theta}\left(\bar{\Delta}_{0, n}\right)=P_{\theta}(\Lambda)=1
$$

Если существует измеримое множество $A \subseteq \Delta_{0}$ такое, что $P_{\theta}(A)>0$, то из соотношения $\Lambda \cap A=\varnothing$ следует, что $P_{\theta}(\Lambda \cup A)=P_{\theta}(\Lambda)+P_{\theta}(A)>1$. Значит, для любого $\theta \in \Theta$ вероятность $P_{\theta}\left(\Delta_{0}\right)=0$.

Рассмотрим $\bar{D}_{0, n}$ и $D_{\theta, n}$. По определению, $P_{\theta, n}\left(D_{\theta, n}\right)=1$; предположим, что множество запретов для последовательности $P_{0, n}$ не пусто. Положим $S_{n}=\bar{D}_{0, n}, \Sigma_{n}=S_{n} \times X^{\infty}$. Для всех $n$ вероятность $P_{0, n}\left(S_{n}\right)=0$. Последовательность $\Sigma_{n}$ не убывает; обозначим ее предел через $\Sigma$. По формуле полной вероятности $P_{\theta}\left(\Delta_{\theta}\right)=P_{\theta}\left(\Delta_{\theta} \cap \Sigma\right)+P_{\theta}\left(\Delta_{\theta} \cap \bar{\Sigma}\right)$. Из построения критических множеств,

$$
\bar{\Sigma}=\overline{\bigcup_{n=1}^{\infty} \bar{\Delta}_{0, n}}=\Delta_{0} .
$$


Кроме того, выполняется равенство

$$
P_{\theta}\left(\Delta_{\theta} \cap \Delta_{0}\right)=P_{\theta}\left(\Delta_{0}\right)-P_{\theta}\left(\bar{\Delta}_{\theta} \cap \Delta_{0}\right) .
$$

В правой части последнего равенства первая вероятность равна 0 по условию, а вторая равна 0 по определению $\Delta_{\theta}$. Отсюда следует, что $P_{\theta}\left(\Delta_{\theta} \cap \Sigma\right)=1$. Поэтому $P_{\theta}(\Sigma)=1$. Отсюда получаем, что

$$
\lim _{n \rightarrow \infty} P_{\theta}\left(\Sigma_{n}\right)=1 .
$$

Это доказывает, что построенная последовательность критериев является состоятельной.

Осталось показать, что в условиях теоремы множество запретов не может быть пустым. Предположим противное. Тогда для каждого $n$ множество $\bar{D}_{0, n}=\varnothing$. Отсюда следует, что для каждого $n$ множество $D_{0, n}=X^{n}$. Переходя к пределу, получим, что $\Delta_{0}=X^{\infty}$. Это противоречит условию теоремы, что для каждого $\theta \in \Theta$ вероятность $P_{\theta}\left(\Delta_{0}\right)=0$. Теорема доказана.

Пример 6. Условия теоремы выполняются, например, когда число точек в множестве $\Delta_{0, n}$, умноженное на максимальную вероятность появления каждой из этих точек в мере $P_{\theta, n}$, стремится к 0 при $n \rightarrow \infty$.

\section{5. Заключение}

1. Алгоритм принятия статистических решений на основе запретов во многих случаях эффективнее традиционных статистических подходов, основанных на обработке накопленных данных с помощью аналогов законов больших чисел.

2. В случае, когда множество запретов ограничено, поиск запретов требует ограниченного времени и ресурсов.

3. Некоторые вероятностно-статистические модели можно упростить с помощью введения запретов, что позволит проще моделировать сложные задачи.

Дальнейшие исследования предполагают построение алгоритмов для нахождения минимального множества запретов; нахождение условий, когда множество запретов конечно; применение статистических процедур, основанных на запретах, в практических задачах.

\section{Список литературы}

1. Грушо А. А., Тимонина Е. Е., Некоторые связи между дискретными статистическими задачами и свойствами вероятностных мер на топологических пространствах. Дискретная математика (2006) 18, №4, 128-135.

2. Неве Ж., Математические основы теории вероятностей. Мир, Москва, 1969.

3. Бурбаки Н., Общая топология. Основные структуры. Наука, Москва, 1968.

4. Прохоров Ю. В., Розанов Ю. А., Теория вероятностей. Наука, Москва, 1993.

5. Grusho A., Grusho N., Timonina E., Problems of modeling in the analysis of covert channels. Lecture Notes Computer Sci. (2010) 6258, 118-124.

6. Simmons G. J., The prisoner's problem and the subliminal channel. In: Advances in Cryptology: Proceedings of Crypto'83 (Chaum D., ed.). Plenum, 1984, pp. 51-67.

7. Леман Э., Проверка статистических гипотез. Наука, Москва, 1964.

Статья поступила 13.12.2010. 